\title{
DIAGNÓSTICO DE ANOMALIAS DENTÁRIAS EM RADIOGRAFIAS PANORÂMICAS
}

\author{
DIAGNOSIS OF ANOMALIES IN DENTAL PANORAMIC X-RAY
}

\section{Gardene Paiva MAGALHÃES1; Evanildo Canuto PAZ; Yara Teresinha Correa Silva SOUSA2; Carla Maria de Carvalho LEITE³; Carlos Alberto Monteiro FALCÃO4; Maria Ângela Arêa Leão FERRAZ ${ }^{3}$.}

1-Discente do curso de Graduação em Odontologia do Centro Universitário UNINOVAFAPI, Teresina, Brasil.

2 - Doutora em Patologia Experimental e Comparada pela Universidade de São Paulo, Brasil.

3 - Doutora em Endodontia pela Universidade de Ribeirão Preto (UNAERP), São Paulo, Brasil.

4 - Doutor em Endodontia São Leopoldo MANDIC, São Paulo, Brasil.

\section{RESUMO}

Introdução: As alterações que ocorrem no processo de odontogênese são denominadas anomalias dentárias e são classificadas quanto à forma, como geminação, fusão, concrescência, dilaceração, cúspide em garra, dens in dente, taurodontia, micro e macrodontia, quanto ao número: dentes ou raízes supranumerários e agenesias e quanto posição, como transmigrações ou ectopias. Objetivo: Determinar a prevalência das anomalias dentárias radiografias de prontuários de pacientes atendidos na clínica de uma Instituição de Ensino Particular. Material e Métodos: Análise de 300 radiografias panorâmicas em negatoscópio, iluminado por duas lâmpadas fluorescentes de 20 watts cada, recoberto com máscara preta, com uma abertura central medindo $15 \times 30 \mathrm{~cm}$ onde o filme panorâmico foi fixado, realizadas em ambiente escuro e, com o auxílio de lupa (aumento 2X) e interpretadas conjuntamente por dois observadores calibrados. Os achados radiográficos foram registrados em tabela própria, constando número prontuário, gênero, presença de alterações e anomalias dentárias de forma, número e posição. A análise estatística descritiva foi realizada pelo cálculo da frequência e percentual de acometimento e relações entre gênero e alterações dentárias encontradas. Resultados: Ocorrência de dilacerações $(3,32 \%)$, dentes cônicos $(1 \%)$, taurodontia $(0,66 \%)$, dens in dente $(0,33 \%)$ e cúspide em garra $(5,66 \%)$, em relação às anomalias de tamanho observaram-se microdontias (5,33\%), nos distúrbios de posição ocorrência como erupção ectópica $(0,33 \%)$ e em casos de alterações de número, dentes $(4,32 \%)$ e raízes supranumerárias $(0,66 \%)$, sem diferenças estatísticas entre os gêneros. Conclusão: A prevalência de variações anatômicas em dentes permanentes é baixa, porém não rara, sem distinção entre os gêneros, mas de significado clínico por induzir, em algumas situações, o comprometimento pulpar ou dificultar terapias odontológicas.

Palavras-chave: Dente; Anormalidades maxilofaciais; Radiografia Panorâmica.

\section{INTRODUÇÃO}

A presença de anomalias dentárias tem sido relatada com frequência na literatura mundial. Condições como geminação, fusão, concrescência, dilaceração, cúspide em garra, dens in dente, taurodontia e raízes supranumerárias são descritas como distúrbios no desenvolvimento da forma dos dentes, enquanto que micro e macrodontia são distúrbios de tamanho, dentes supranumerários e anodontias são alterações no número de dentes ${ }^{1}$. Anomalias de posição são dentes localizados em local diferente do desenvolvimento original, como transmigrações ou ectopias².

Estas anomalias são causadas por distúrbios no processo de morfodiferenciação durante a odontogênese, tendo os fatores hereditários e genéticos como predisponentes ao desenvolvimento da alteração ${ }^{3}$ e por alterações metabólicas, mutações, fatores ambientais, biológicos ou por combinação de vários fatores ${ }^{4}$, com a possibilidade de múltiplas alterações em um mesmo paciente ${ }^{5}$.

A ocorrência destes distúrbios é relatada na literatura podendo também ocorrer como parte de síndromes ${ }^{6}$, assim como dentes decíduos e dentes permanentes podem ser afetados ${ }^{7}$.
A incidência e grau de expressão destas alterações de desenvolvimento podem fornecer informações importantes para estudos de genética e filogenética e ajudar a compreender variações dentro e entre populações ${ }^{4}$. A identificação destas alterações antes do estabelecimento de patologias deve ser considerada como medida preventiva para proservação ou instituição de medidas conservadoras a fim de se evitar o desenvolvimento de patologias de ordem local ou geral ${ }^{8}$.

A prevalência de anomalias dentárias de desenvolvimento em diferentes populações e grupos étnicos é tema de estudos, com diferenças atribuídas a padrões raciais, amostragem técnicas e diferentes critérios de diagnóstico ${ }^{4}$.

Estas alterações são usualmente diagnosticadas em exames radiográficos de rotina ou nos exames que precedem tratamento ortodôntico ${ }^{9}$. Podem apresentar-se de forma assintomática ${ }^{8}$ ou provocar alteração na função mastigatória por interferência oclusal e danos estéticos ${ }^{10}$ e podem, ainda, causar patologias de ordem local e sistêmica ${ }^{11,12}$.

O objetivo deste trabalho é determinar a prevalência e a 
frequência de alterações dentárias de forma (geminação, fusão, concrescência, dilaceração, cúspide em garra, dens in dente, taurodontia), tamanho (micro e macrodontia), número (raízes e dentes supranumerários) e posição (transmigração e ectopias) em radiografias panorâmicas e a variação entre os gêneros.

\section{MATERIAL E MÉTODOS}

Este estudo retrospectivo foi realizado utilizando-se 300 radiografias panorâmicas, tomadas no período de 2009 a 2011 por indicações clínicas diversas, selecionadas de forma aleatória e aprovado pelo Comitê de Ética sob o número 02727812.7.0000.5498. Os critérios de seleção dos prontuários incluíram a ficha estar preenchida completamente e conter radiografia panorâmica em adequado padrão técnico.

A análise das radiografias panorâmicas foi realizada em negatoscópio, iluminado por duas lâmpadas fluorescentes de 20 watts cada, recoberto com máscara preta, com uma abertura central medindo $15 \times 30 \mathrm{~cm}$ onde o filme panorâmico foi fixado. As interpretações foram realizadas em ambiente escuro e, com o auxílio de lupa (aumento 2X), e interpretadas conjuntamente por dois observadores calibrados.

Os achados radiográficos foram registrados em tabela própria, confeccionada para este fim, constando: número prontuário, data nascimento, gênero, presença de alterações e anomalias dentárias de forma, tamanho, número e posição (geminação, fusão, concrescência, dilaceração, cúspide em garra, dens in dente, taurodontia, micro e macrodontia, raízes e dentes supranumerários transmigração e ectopias).

A análise estatística foi descritiva através do cálculo da frequência e percentual de acometimento e relações entre gênero e alterações dentárias encontradas. A avaliação de significância foi realizada pelo teste qui quadrado, com nível de significância de $5 \%(p<0,05 \%)$ com emprego do programa SPSS.

\section{RESULTADOS}

Das 300 radiografias interpretadas, 43,33\% apresentaram alterações dentárias. Houve ocorrência de anomalias de forma tais como dilaceração (7 pré-molares inferiores e 3 terceiros molares inferiores), dentes conoides (2 incisivos laterais), taurodontia (2 molares numa mesma imagem radiográfica), um dens in dente em um canino superior e cúspide em garra em incisivos superiores.

Em relação às anomalias de tamanho observou-se microdontias em terceiros molares superiores, em um terceiro molar inferior e em um segundo molar superior e nos distúrbios de posição e ocorrências como posição ectópica de um terceiro molar superior (Figura 1). As alterações de número observadas foram dentes supranumerários, sendo 5 molares e 8 pré-molares (Figura 2) e raízes supranumerárias em 2 caninos inferiores, conforme Tabela 1.

Pela análise estatística realizada através do Teste qui quadrado não foi observado diferença estatística entre os gêneros.

\section{DISCUSSÃO}

Anomalias dentais são achados relativamente comuns, e diagnosticados em exames de rotina. São relacionadas a fatores genéticos e ambientais ${ }^{4}$. A porcentagem de alterações em 43,33\% das radiografias analisadas corresponde com resultados de Uslu,

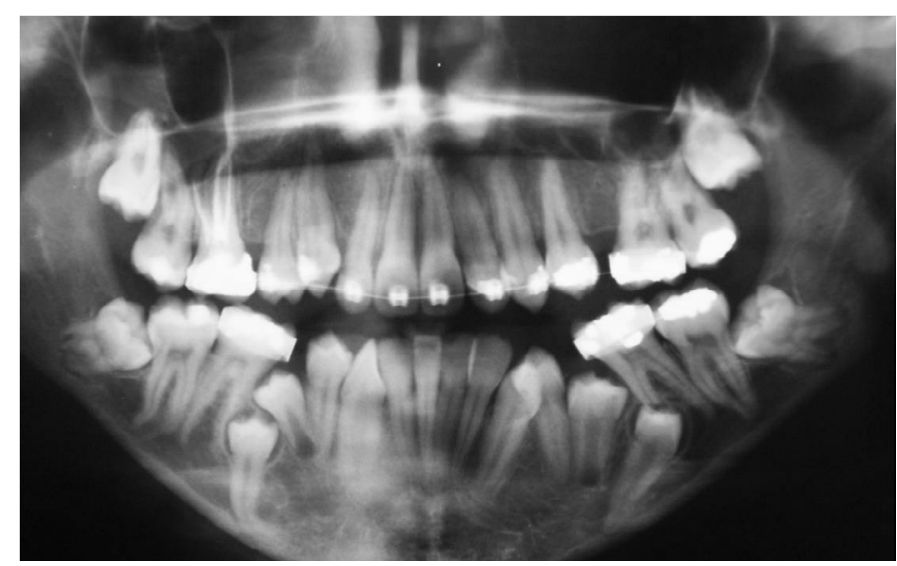

Figura 1 - Terceiro molar superior em posição ectópica

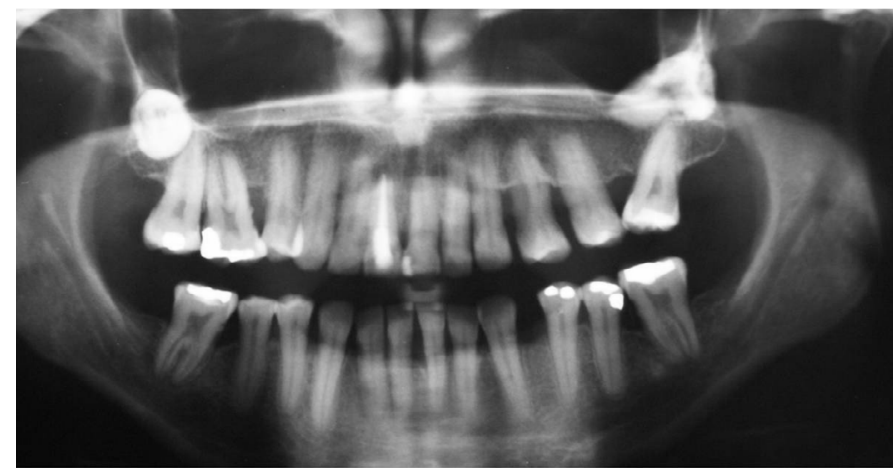

Figura 2 - Pré-molares supranumerários

Tabela 1 - Distribuição anomalias de forma

\begin{tabular}{|c|c|c|c|c|}
\hline ANOMALIAS & TIPO & $\begin{array}{l}\text { GÊNERO } \\
\text { FEMININO(\%) }\end{array}$ & $\begin{array}{l}\text { GÊNERO } \\
\text { MASCULINO(\%) }\end{array}$ & $\begin{array}{l}\text { TOTAL } \\
(\%)\end{array}$ \\
\hline \multirow{5}{*}{ FORMA } & DILACERAÇÃO & $08(2,66)$ & $2(0,66)$ & $10(3,32)$ \\
\hline & CÔNICO & $03(1)$ & 0 & $03(1)$ \\
\hline & TAURODONTIA & $02(0,66)$ & 0 & $02(0,66)$ \\
\hline & DENS IN DENTE & $01(0,33)$ & 0 & $01(0,33)$ \\
\hline & $\begin{array}{l}\text { CÚSPIDE EM } \\
\text { GARRA }\end{array}$ & $10(3,33)$ & $07(2,33)$ & $17(5,66)$ \\
\hline \multirow{2}{*}{ TAMANHO } & MACRODONTIA & 0 & 0 & 0 \\
\hline & MICRODONTIA & $10(3,33)$ & $06(2)$ & $16(5,33)$ \\
\hline POSIÇÃO & ECTOPIA & 0 & $1(0,33)$ & $1(0,33)$ \\
\hline \multirow{2}{*}{ NÚMERO } & $\begin{array}{l}\text { DENTE } \\
\text { SUPRANUMERÁRIO }\end{array}$ & $5(1,66)$ & $8(2,66)$ & $13(4,32)$ \\
\hline & $\begin{array}{l}\text { RAIZ } \\
\text { SUPRANUMERÁRIA }\end{array}$ & $1(0,33)$ & $1(0,33)$ & $2(0,66)$ \\
\hline
\end{tabular}

Akcam, Evirgen e Cebeci ${ }^{13}$ (2011) que relatam 40,3\% de anomalias encontradas em pacientes com maloclusões pela análise de radiografias panorâmicas, diferem, no entanto, dos resultados de Kazanci, Celikoglu, Miloglu, Ceylan e Kamak (2011) ${ }^{4}$ que 
encontraram apenas $14,28 \%$ de alterações em 3165 pacientes ortodônticos analisados através de radiografias panorâmicas, fotografias intraorais e modelos de gesso.

O diagnóstico das alterações e/ou anomalias encontradas clínica ou radiograficamente é necessário para o sucesso do plano de tratamento odontológico em todas as especialidades odontológicas, minimizando assim estresse e custos aos pacientes ${ }^{14}$.

A radiografia panorâmica torna-se relevante para o diagnóstico precoce de condições patológicas, por se tratar de exame complementar mais acessível à população e facilitar a terapêutica pela possibilidade de visão do complexo maxilomandibular de forma bilateral em um único filme ${ }^{14}$.

Nesta pesquisa, os dentes que apresentaram dilaceração foram pré-molares e terceiros molares inferiores, na literatura, os dentes citados como de maior prevalência são os pré-molares seguidos dos dentes anteriores ${ }^{14}$, em pesquisa da prevalência de dilaceração por meio de radiografias panorâmicas na população turca ${ }^{15}$ e por meio de radiografias periapicais na população nigeriana o maior número de dentes com esta variação foram os dentes posteriores ${ }^{16}$.

A etiologia mais comum para dilaceração, em dentes anteriores, refere-se a trauma no antecessor decíduo ${ }^{17}$, embora alguns achados não indiquem esta relação nos dentes posteriores. A baixa prevalência encontrada pode estar associada com o fato da radiografia panorâmica não ser o exame de imagem mais indicado para diagnóstico desta variação anatômica por não evidenciar imagens nas direções bucal e palatal/lingual ${ }^{15}$.

Dentes cônicos são comumente evidenciados em incisivos laterais e em mulheres ${ }^{18}$ concordando com o estudo.

A frequência de molares com alteração de forma taurodontia encontrada foi inferior quando comparada ao outros estudos: Burklein, Breuer, Schafer ${ }^{6}$ (2011) relataram 2.25\% desta alteração em 800 radiografias periapicais em pacientes de até 18 anos. $\mathrm{O}$ limite de idade dos pacientes pode justificar esta diferença de ocorrência de taurodontia.

Dens in dente apresenta importância clínica por induzir a comprometimento pulpar $^{19}$. Em estudo envolvendo 1.123 pacientes apenas $2,4 \%$ de prevalência foi evidenciada, confirmando baixa incidência desta anomalia de forma ${ }^{20}$.

Elementos dentários com cúspide em garra foram considerados quando houvesse uma estrutura radiopaca em forma de V sobreposta à estrutura do dente; Hamasha e Safadi $(2010)^{21}$ encontraram 0,55\% em 3.024 radiografias periapicais, neste estudo evidenciaram-se valores acima do encontrado na literatura (5,66\%), observando-se características peculiares em cada população estudada.

Em pesquisa com 533 radiografias panorâmicas ${ }^{22}$, encontraram $0,75 \%$ de macrodontias em ambos os gêneros; considerando o baixo percentual de prevalência da condição e o tamanho da amostra analisada, não parece incoerente a ausência de registro de macrodontias nas radiografias analisadas neste estudo.

Um maior percentual de microdontias foi observado ${ }^{22}, 2,81 \%$; em contraponto com este trabalho onde relatamos 5,33\% de microdontias, diferindo também a prevalência nos dentes envolvidos: incisivo lateral superior e terceiro molar superior, respectivamente.

Erupção ectópica é considerada anomalia de acometimento raro $^{23}$. Concordando com a baixa incidência encontrada (um terceiro molar superior no ramo ascendente da mandíbula), registro de 1,52\% de ectopias em 3165 radiografias panorâmicas, com maior incidência de segundo molares inferiores já foi descrito ${ }^{4}$.

A presença de 4,32\% de dentes supranumerários encontra-se em desacordo com literatura, em estudo com o mesmo tipo de imagens radiográficas, em pacientes ortodônticos, relatou frequência de 1,33\% de supranumerários ${ }^{4}$. Este fato pode ser relacionado ao fato das imagens utilizadas neste estudo serem de arquivo de Instituição de Ensino, que configura referência para encaminhamento de patologias e variações de anatomia, a fim de receberem tratamento adequado.

A presença de raízes acessórias ou supranumerárias é um acometimento raro, mas seu significado é essencial para sucesso de tratamentos empregados seja tratamento pulpar ou extração de dentes, pois podem representar dificuldades clínicas especialmente quando elas não estão previstas ${ }^{20}$. Tanto dentes decíduos quanto os permanentes podem apresentar esta variação ${ }^{23}$ e baixa prevalência ${ }^{24}$.

\section{CONCLUSÃO}

Pela análise dos dados coletados podemos concluir que a prevalência de variações anatômicas em dentes permanentes é baixa, porém não rara, sem distinção entre os gêneros, mas de significado clínico por induzir, em algumas situações, o comprometimento pulpar ou dificultar terapias odontológicas.

\section{REFERÊNCIAS}

01. Shafer WG, Hine MK, Levy BM. Tratado de Patologia Bucal Distúrbios do Desenvolvimento e do Crescimento. Rio de Janeiro: Guanabara; 1987. p. 33-46.

02. Nascimento FFAO, Silva CJ, Marquez IM, Magalhães AEO. Transmigração de Caninos Inferiores. BCI. 2001; 30(8): 165-167.

03. Sarraf-Shirazi A, Rezaiefar M, Forghani MA. Rare Case of Multiple Talon Cusps in Three Siblings. Braz Dent J. 2010; 21(5): 463-466.

04. Kazanci F, Celikoglu M, Miloglu O, Ceylan I, Kamak H. Frequency and distribution of developmental anomalies in the permanent teeth of a Turkish orthodontic patient population. Journal of Dental Sciences. 2011; 6(2):82-89.

05. Rao YG, Guo LY, Hu T. Multiple Dens Evaginatus of Premolars and Molars in Chinese Dentition. Int J Oral Sci. 2010; 2(3): 177-180.

06. Burklein S, Breuer D, Schafer E. Prevalence of Taurodont and Pyramidal Molars in a German Population. J Endod. 2011;37(2):158-162.

07. Guttal KS, Naikmasurb VG, Bhargavac P, Bathid RJ. Frequency of Developmental Dental Anomalies in the Indian Population. Eur J Den. 2010; 5(4): 263-69.

08. Demartis P, Dessı C, Cotti M, Cotti E. Endodontic Treatment and Hypotheses on an Unusual Case of Dens Invaginatus. J Endod. 2009; 35(3):417-421.

09. Kang BC, Yoon SJ, Lee JS, Al-Rawi W, Palomo JM. The Use of Cone Beam Computed Tomography for the Evaluation of Pathology, Developmental Anomalies and Traumatic Injuries Relevant to Orthodontics. Sem Orthod. 2011; 17(1):20-33.

10. Nagaveni NB, Umashankara KV, Sreedev RBP, Radhika NB, Satisha TS. Multi-Lobed Mesiodens with a Palatal Talon Cusp. A Rare Case Report. Braz Dent J. 2010; 21(4): 375-378.

11. Ababneh HT, Taha AH, Abbadi MS, Karasneh JA, Khader YS. The association of aggressive and chronic periodontitis with systemic 
manifestations and dental anomalies in a jordanian population: a case control study. Head Face Med. 2010; 6(30): 1-8.

12. Arsenault M, Anderson RD, Dyment H, Macellan J, Doyle T. Facial Cellulitis Secondary to Dens Invaginatus: A Case Report. J Can Dent Assoc. 2010; 76(a114): 40-3.

13. Uslu O, Akcam MO, Evirgen S, Cebeci I. Prevalence of dental anomalies in various malocclusions. Am J Orthod Dentofacial Orthop. 2009; 135(3):328-35.

14. Armond MC, Saliba JHM, Silva VKS, Jaqueira LMF, Generoso R, Ribeiro A, Borges DD, Paiva AM. Prevalência de Alterações Dentárias em Crianças de 2 a 13 Anos de Idade. Pesq Bras Odontoped Clin Integr. 2008; 8(1):69-73.

15. Miloglu O, Cakici F, Caglayan F, Yilmaz A, Demirkaya F. The prevalence of root dilacerations in a Turkish population. Med Oral Patol Oral Cir Bucal. 2010; 15(3):e441-4.

16. Udoye CI, Jafarzadeh H. Dilaceration among Nigerians: prevalence, distribution, and its relationship with trauma. Dental Traumatology. 2009; 25(4): 439-441.

17. Tewari N, Pandey RK. Multiple abnormalities in permanent maxillary incisors following trauma to the primary dentition. J Indian Soc Pedod Prev Dent. 2011; 29(2): 161-4.

18. Wu H, Feng HL. A survey of number and morphology anomalies in permanent teeth of 6453 youths between 17 to 21 years old. Estomatologia. 2005; 40(6):489-90.

19. Zengin AZ, Sumer AP, Celenk P. Double Dens Invaginatus: Report of Three Cases. European Journal of Dentistry. 2009; 3(1): 67-70.

20. Gupta SK, Saxena P, Jain S, Jain D. Prevalence and distribution of selected developmental dental anomalies in an Indian population. J Oral Sci. 2011; 53(2):231-8.

21. Hamasha AAH, Safadi RA. Prevalence of talon cusps in Jordanian permanent teeth: a radiographic study. BMC Oral Health. 2010; 10:6.

22. Girondi JR, Fenyo-Pereira M, Campos PSF, Panella J. Estudo da prevalência das anomalias dentárias de desenvolvimento em dada população, com o uso de radiografias panorâmicas. Revista de Odontologia da Universidade Cidade de São Paulo. 2006; 18(1): 15-21.

23. Gupta S, Nagaveni NB, Chandranee NJ. Three-rooted mandibular first primary molar: Report of three cases. Contemp Clin Dent. 2012; 3(Suppl1):S134-S136.

24. Mohan S, Kankariya H, Harjani B, Sharma H. Ectopic third molar in the maxillary sinus. Natl J Maxillofac Surg. 2011; 2(2): 222-224.

25. Ahmed HM, Abbott PV. Accessory roots in maxillary molar teeth: a review and endodontic considerations. Aust Dent J. 2012; 57(2):123-31.

\section{ABSTRACT}

Introduction: The changes that occur in the process of odontogenesis are called dental anomalies and are classified according to form as twinning, fusion, concrescence, laceration, claw cusp, dens in dente, taurodontia, micro and macrodontia, the number: teeth or agenesis and supernumerary roots and the position as transmigration or ectopic. Objective: To determine the prevalence of dental anomalies radiographs records of patients seen at the clinic of a private education institution. Material and Methods: Analysis of 300 panoramic radiographs in light box illuminated by two fluorescent lamps of 20 watts each, coated with black mask, with a central opening where the measuring $15 \times 30 \mathrm{~cm}$ panoramic film was set, held in dark, with the aid of magnifying ( $2 \mathrm{X}$ increase) and interpreted jointly by two calibrated observers. The radiographic findings were recorded in the table itself, consisting chart number, gender, presence of dental anomalies and changes in shape, number and position.
Descriptive statistical analysis was performed by calculating the frequency and percentage of involvement and relationships between gender and dental abnormalities found. Results: Occurrence of lacerations $(3.32 \%)$, conical teeth $(1 \%)$, taurodontia $(0.66 \%)$ in tooth dens $(0.33 \%)$ and peak claw $(5.66 \%)$ in relation to size abnormalities were observed microdontia (5.33 $\%)$, the occurrence position disorders such as ectopic eruption $(0.33 \%)$ and number of cases of changes teeth $(4.32 \%)$ and roots supernumerary $(0.66 \%)$, without statistical differences between genders. Conclusion: The prevalence of anatomical variations in permanent teeth is low, but not rare, without gender distinction, but to induce clinical significance, in some situations, the pulp involvement or hinder dental therapies.

Keywords: Tooth; Anormalities Maxillofacial; Panoramic Radiography.

\section{AUTOR PARA CORRESPONDÊNCIA}

Gardene Paiva Magalhães

Endereço: Rua Dom Pedro II, n 450 - Centro - Altos - Piauí, CEP 64290-000

Telefone +55 86 99437-1920

Email: gardenemagalhaes7@gmail.com 